\title{
An active approach in the treatment of post-concussion syndrome - Evidence-based practice in a collective case study
}

\author{
Thomas Christensen ${ }^{1}$, Marianne Kyvsgaard ${ }^{1}$, Peter Vindelev ${ }^{2}$, Sofie Joergensen ${ }^{3}$, and bodil \\ wiberg larsson ${ }^{1}$ \\ ${ }^{1}$ University College Copenhagen - Carlsberg Campus \\ ${ }^{2}$ Rigshospitalet \\ ${ }^{3}$ Bispebjerg Hospital
}

July 7, 2020

\begin{abstract}
Rational, Aims and Objectives: Prolonged symptoms after a mTBI, known as Post-Concussion Syndrome (PCS), remains a challenging area of rehabilitation. Evidence shows that an active approach can improve prognosis, however PCS is a multifaceted condition with many comorbidities and large variety in patient response. This study investigated the use of submaximal aerobic exercise and body awareness therapy, and the influence on symptoms in PCS cases, viewed through the lens of evidence-based practice (EBP). Method: Four cases were separated into two case studies. Cases in Study A received an aerobic exercise protocol consisting of 8 individual sessions distributed twice a week over 4 weeks, with additional cervical endurance training. Cases in Study B received a body awareness therapy protocol consisting of 6 guided practices distributed twice a week over 3 weeks. Using method triangulation, both quantitative and qualitative data were gathered through the use of the Rivermead PostConcussion Questionnaire (RPQ) and semi-structured interviews, as well as the Craniocervical Flexion Test (CCFT) in Study A, and the Short Form-36v2 (SF-36) questionnaire in study B. Lastly, research evidence on PCS was included to contextualize cases. Results: Both cases in Study A showed marked improvements in their RPQ scores, but only one showed improvement in the CCFT. In Study B, one case improved in RPQ score and in the mental component of SF-36v2, while the other case did not experience any significant change. All cases expressed positive associations with the interventions during the interviews. Conclusion: These results illustrate how an active and individualized approach can represent important qualities that can be applied to further and larger studies. Based on the results and discussion of this paper, relevant findings and suggestions are summarized in a modified EBP model, which may be of help to practitioners in dealing with PCS patients in the clinical practice.
\end{abstract}

\section{Keywords}

Mind Body Therapy, Mild Traumatic Brain Injury, Aerobic Exercise, Autonomic Nervous System, Physical Therapy Modalities, Neck

\section{Introduction}

In Denmark approximately 25.000 cases of mTBI are diagnosed each year ${ }^{1}$ with an additional estimated $25 \%$ undiagnosed, ${ }^{2}$ bringing the national incidence rate up to 545 pr. 100.000 . This is in line with the international rate where incidents of mTBI, including diagnosed and undiagnosed, are estimated to be about 600 per 100.000 worldwide, or around 45 million a year. ${ }^{3}$ Of these, roughly $75-90 \%$ experience gradual remission of symptoms within 14 days, whereas $10-25 \%$ continue having symptoms, which may then devolve into Post-Concussion Syndrome (PCS). ${ }^{4,5}$ While no consensus on the timeframe exists, PCS can be defined as 
experiencing persistent symptoms of a mTBI for longer than 3 months, as by the DSM- 5 criteria, with additional psychosocial sequelae. ${ }^{6,7}$ Patients often experience difficulties in maintaining their social, professional and physical levels of activity, and may be at risk of not participating in and contributing to the financial state of society. A Danish survey found that $19 \%$ of all mTBI patients were on sick leave for more than a month and that $2 \%$ had still not returned a year after the initial trauma. ${ }^{8}$ In the U.S., a report from the Agency for Health Research and Quality found that TBI patients across all levels of severity had a higher prevalence of long term depression at $30 \%$, which is more than three times the national average at $8-10 \%$, as well as higher rates of anxiety and PTSD. ${ }^{9}$

An increasing amount of evidence shows that an active approach, contrary to earlier beliefs about a "wait and see approach", can improve outcomes and long term prognosis. ${ }^{10-12}$ The international consensus statement on Concussion in Sports from 2016 in Berlin recommends a period of 24-48 hours of physical and cognitive rest after the initial trauma, followed by a progressively active rehabilitation. ${ }^{13}$ It is well established that PCS patients can experience barriers towards physical and cognitive activity, with higher levels of cognitive exertion associated with longer duration of symptoms in some, ${ }^{14}$ and that adequate management between rest and activity is a central yet complex part of the recovery process. ${ }^{15-17}$ These barriers often manifest as exercise intolerance, cervicogenic headaches, rapid eye exhaustion, dizziness, nausea, excessive fatigue, and a low threshold for managing sensory input. ${ }^{18-22}$ Therefore, it is important to clarify the parameters of an active approach to treatment in a rehabilitation setting. In this context, an active approach can be both an up or downregulation of physical and mental stimulation, as well as providing education and tools to promote patient self-efficacy.

One of the major challenges in dealing with PCS is categorizing the underlying pathologies and understanding their effect on the symptomatology, to which the evidence suggests a multitude of neurophysiological and psychosomatic mechanisms. These mechanisms include disruptions in cerebral blood flow (CBF), ${ }^{23,24}$ changes in brain metabolism,${ }^{25}$ axon damage, ${ }^{26}$ changes in autonomic and neuroendocrine function, ${ }^{27-30}$ cervical injury and postural weakness, ${ }^{31}$ disturbances in the vestibular and oculomotor system, ${ }^{32,33}$ and neuropsychological dysfunctions. ${ }^{34,35}$ These myriad of components and overlapping comorbidities adds to the complexity of PCS, and highlights the difficulties of having a single treatment regimen. It may also explain the varying responses to clinical assessment. ${ }^{36}$

A useful tool in organizing and providing the most relevant care is the evidence-based practice (EBP) model originally coined by Sackett ${ }^{37}$ and further developed through Straus ${ }^{38}$ and Howick. ${ }^{39}$ While different iterations exists, the model formulates three dimensions of best practice: Best Research Evidence which is the highest graded and most appropriate evidence in the scientific literature, Clinical Expertise which is the clinical experience and knowledge, and Patient Preferences which is the individual patient preferences, their values or larger group characteristics. Using the EBP model practitioners can more easily stratify the individual variables and formulate a cohesive plan of action to target the multifaceted nature of PCS.

By applying two distinct interventions, this paper will examine how an active and individualized approach can be used in the treatment of PCS. The empirical data will be derived from two case studies, each consisting of two cases with separate protocols, highlighting the aspects of overcoming symptoms of PCS with insight from the cases, as well as an inclusion of the relevant literature. This will be viewed through the lens of a modified EBP model and ultimately summarized in a visual presentation, to more appropriately transfer knowledge to the clinical practice.

\section{Methodology}

In both case studies method triangulation was used as the foundation of the study designs, including a quantifying positivistic approach to measure changes in clinical symptoms as a result of the interventions, combined with a qualitative humanistic approach used to gain an understanding of the cases' subjective experiences of the interventions. The quantitative data was gathered through clinical examination and 
questionnaires, and the qualitative understanding was gathered through the use of semi-structured interviews. Because of the volatile symptoms of PCS, the interventions were designed as a cyclical process with the intention of including cases by listening to their feedback and adjusting protocols, if necessary, based on any potentially adverse reactions. This design was inspired by Malterud's research spiral which is based on a continuous evaluation and defining of the problem and intervention, thus preparing researchers to be adaptable and increase transferability to the rehabilitation. ${ }^{40,41}$

\subsection{Ethical Foundation}

Permission for initiating the study was granted from The Danish National Committee on Health Research Ethics prior to the beginning of the project, concluding that cases were not at risk of harm, and the ethical aspects of the project were assessed according to the Helsinki Declaration. ${ }^{42}$ Cases were given oral and written information about the protocols and signed an informed consent form with the right to withdraw at any time. All cases were anonymized, and qualitative data collection was handled with discretion with no sharing of personal information.

\subsection{Case and Study Descriptions}

Case characteristics are presented in Table 1 and 2. The four cases were recruited through a collaboration with a center of rehabilitation in the capital region of Denmark and separated into two different case studies, Study A and Study B, examining two different intervention protocols. Cases in Study A participated in a submaximal graded aerobic exercise intervention supplemented by postural correction of the cervical spine and cases in Study B participated in a modified body awareness intervention with additional group feedback sessions.

\section{TABLE 1}

Study A Inclusion criteria; Persistent symptoms of mTBI for more than 12 months following initial trauma, above the age of 18 and able to sit on a stationary bike without referred pain.

Study B inclusion criteria; Persistent symptoms of mTBI for more than 12 months following initial trauma, above the age of 18 , able to stand for a duration up to 20 minutes, able to lie flat on their backs.

Excluding criteria; Unable to communicate in Danish, unable to attend twice a week and contraindicated disorders making the planned interventions impossible.

\section{TABLE 2}

\subsection{Data and Analysis}

All four cases underwent a clinical examination before the interventions covering relevant injury history, current and previous work history, social participation, daily activities and bodily functions in relation to the International Classification of Functioning Disability and Health (ICF) model. ${ }^{43}$ All cases were scored on the Rivermead-Post Concussion Questionnaire (RPQ), a standardized post-concussion questionnaire with two subscores ${ }^{44}$ pre- and post-intervention, as well as a semi-structured qualitative interview post intervention. Additionally, cases in Study A underwent a Craniocervical Flexion Test (CCFT) ${ }^{45}$ pre and post intervention, and cases in Study B were scored on the SF-36v2 Quality of Life (QOL) questionnaire ${ }^{46}$ pre and post intervention. RPQ, SF-36 and CFFT have shown to be valid testing tools in measuring prolonged symptoms of mTBI, QOL and cervical function in different population groups with moderate to high construct validity and reliability. ${ }^{47-50}$ Questionnaires followed the guidelines for analysis of $\mathrm{RPQ}^{47}$ and SF-36v2 $2^{51,52}$ using simple comparative statistics in calculating averages and the semi-structured interviews were recorded, transcribed and analysed by identifying codes and sub-codes, which were clustered ad modum Kvale. ${ }^{53}$ The CFFT was administered as per standardized guidelines. ${ }^{45}$ 


\subsection{Interventions}

\section{Study A}

\section{Submaximal Graded Aerobic Exercise}

The aerobic exercise was done on a stationary bike to reduce exposure of cervical compression and impact forces, minimizing symptom exacerbation due to something other than the exercise itself. During the initial session, cases were instructed to reach a baseline heart rate (HR) above 100 BPM if possible, while being monitored for any increase in symptoms which would terminate the test. In each of the following sessions cases then attempted to increase their HR by $10 \mathrm{BPM}$, on the condition that symptoms were not aggravated. HR thresholds were noted during the first and last session. The intervention consisted of 8 individual consultations distributed twice a week over 4 weeks, with the exercise lasting 15 minutes each session.

\section{Correction of malalignment}

Cases were given home exercises based on findings in the CCFT test and posture analysis following Panjabi. ${ }^{54}$ These consisted of low intensity endurance training of the deep cervical flexors with a focus on muscle control and close attention not to activate any superficial muscles, as well as additional static stretching. Exercises were done daily in 1-3 sets of 10 repetitions and stretches were held for 45 seconds up to 3 times a day.

\section{Study B}

\section{Body Awareness Therapy}

The series of movements were inspired by Basic Body Awareness Therapy (BBAT) and designed in cooperation with a physical therapist specialized in BBAT. Large cervical rotations and strenuous movements were removed to minimize possible symptom exacerbation. Cases were guided in the key principles, which emphasize that all movements should be performed with a physical and cognitive presence, allowing them to stay grounded, breathe freely and move energy-economically, in order to make the series flow relaxed and naturally. ${ }^{55,56}$ All sessions were concluded with patients doing a brief body scan lying supine on the floor, followed by a short group feedback session. The intervention consisted of 6 guided practices distributed twice a week over 3 weeks, with each session lasting 45 minutes.

\section{Results}

\subsection{Study A}

\section{Rivermead Post-Concussion Questionnaire}

As shown in Fig. 1 and 2, Case 1 had a reduction from 12 to 8 (33\%) in RPQ-3 score and 44 to 25 (43\%) in RPQ-13 score, pre- to post-intervention. Case 2 had a similar reduction in both scores, going from 5 to 3 (40\%) in RPQ-3 score and 22 to 13 (41\%) in RPQ-13 pre- to post-intervention.

\section{FIGURE 1}

\section{FIGURE 2}

\section{CCFT and Aerobic Threshold}

Case 1 remained a positive grade 3 in the CCFT throughout the study. Case 2 was initially scored as a positive grade 1 in the CCFT, managing the requirements with shaky and uneven movements, but improved to a positive grade 3 post intervention.

As shown in Fig. 3, Case 1 managed a working HR of 100 during the first session and 126 in the last session. The exercise intensity, as a percentage of a calculated maximum HR, went from $55 \%$ in the first session to $70 \%$ in the last session. Case 2 managed a working HR of 119 during the first session and 138 in the last 
session. The exercise intensity went from $73 \%$ in the first session to $85 \%$ in the last session. Both cases experienced no aggravation of symptoms during the exercise.

FIGURE 3

\section{Semi-Structured Interview}

As shown in Table 3, emerging from these codes were themes of protection against overstimulation and the importance of being in a comfortable setting. Cases explained how this was helpful in preventing them from reaching their mental or physical symptom thresholds. Considering their reduced tolerance to sensory input, controlling the surroundings lowered unwanted stimuli and allowed cases to devote their energy to the intervention itself, without dealing with unnecessary triggers. Both cases described an initial feeling of a lifeless body and how they had avoided exercise for a long time before the intervention. Strenuous physical activity can lead to symptom exacerbation in many PCS patients causing a negative feedback loop further reducing physical activity, making their bodies less resilient long term. After the intervention however, both cases expressed feelings of physical success and improved physical confidence which enabled them to continue to exercise on their own.

\section{TABLE 3}

\subsection{Study B}

\section{Rivermead Post-Concussion Questionnaire}

As shown in Fig. 4 and 5, RPQ-3 score remained unchanged for Case 3 from pre- to post-intervention, however there was an increase from 28 to 33 (15\%) in RPQ-13 score. Case 4 had a reduction in both scores, from 5 to 4 (20\%) in RPQ-3 score and 28 to 19 (32\%) in RPQ-13 score, pre- to post intervention.

\section{FIGURE 4}

FIGURE 5

\section{Short Form-36}

As shown in Fig. 6, Case 3 had an increase in the physical component from 30 to 32 (7\%) and the mental component from 37 to $40(8 \%)$ from pre to post intervention.

Case 4 had a decline from 40 to $39(3 \%)$ in the physical component, while the mental component increased, going from 32 to $50(56 \%)$ with a notable improvement on questions regarding levels of concentration and fatigue.

\section{FIGURE 6}

\section{Semi-Structured interview}

As shown in Table 4, emerging from these codes were the meaningfulness of engaging in an acknowledging social space with others, and the positive experience in meeting peers with the same condition and similar challenges. Cases explained how they had withdrawn from social activities due to symptoms and had experienced a loss in the ability to nurture social relationships. Both cases highlighted that attending the intervention had given them a sense of purpose and something to look forward to during the week, which they emphasized was of great importance. Here cases expressed their views on body awareness therapy as a treatment tool, and the pros and cons of this type of activity compared to other more familiar types, like aerobic exercise. Both cases experienced some difficulty conceptualizing the idea initially, which Case 3 continuously had issues with throughout the intervention. They also expressed an improved ability to detect their bodily sensations and Case 4 especially considered this a tool to help reduce unwanted tension and improve self-efficacy. The final codes revolved around reflecting on the intervention and improving ideas to apply to other protocols in the future, as expressed by the cases. The cases underscored the importance of the weekly group feedback session and conversations after each session, which was done with a focus on 
progression and daily strategies, and how this had helped create a constructive environment. Lastly, the cases expressed that a longer intervention duration would be needed for participants to feel fully comfortable in each other's presence, and to improve one's own presence of body and mind during the movements.

TABLE 4

\section{Discussion}

PCS patients are characterized by their heterogeneity and the syndrome is in many ways used as an umbrellaterm for several complex and overlapping physiological and psychological pathologies. When examining the cases we found that, while having had similar experiences, they had different primary symptoms, different concerns and varying degrees of emotional and physical debilitation. Some researchers have argued that dividing PCS into various subcategories, or so called 'post-concussion disorders', could be used to improve outcomes and better treat underlying causes. ${ }^{28,57-59}$ Others have suggested the use of the RPQ, with additional questionnaires, as a possible classification system. ${ }^{60}$ While categorizing the cause and effect relationships remains important in advancing the treatment of PCS, the exact mechanisms between symptomatology and pathology is still unclear. Bearing this in mind, there is a need for a rehabilitation that is multifaceted, explorative and tailored to the individual. ${ }^{4}$ To further reflect on this process, the following section will examine the nature of PCS pathology and rehabilitation through the lens of the EBD model, while contextualizing our cases.

\subsection{Best Research Evidence}

Dysfunction of the ANS, or dysautonomia, acutely following a mTBI has been linked to symptoms of exercise intolerance, lowered tolerance to mental and physical stimuli, decreased cognitive ability, increased stress response and emotional dysfunction. ${ }^{28,61,62}$ Prolonged dysautonomia is prevalent in the PCS group, with a recent review finding at least one form of autonomic dysregulation in the majority of patients. ${ }^{27}$ Evidence shows that the ANS plays a central role in psychological well-being ${ }^{63-65}$ and that declining mental health after a mTBI is unfortunately a common occurrence, ${ }^{35,66}$ with PCS patients demonstrating higher incidence rates of both depression and anxiety compared to controls. ${ }^{67,68}$ Our cases reported an inability to deal with large crowds and various degrees of anxiety, frustration, worry or depression as well as reduced tolerance to external stimuli contributing to social isolation. This is in line with what is often observed in the clinical practice where many PCS patients will abstain from social activities to prevent symptom exacerbation. This abstinence functions as a negative feedback loop creating further withdrawal and worsening symptoms. Considering that prolonged social isolation is linked to poor mental health outcomes in the general population ${ }^{69,70}$ it is not entirely clear to what extent the relationship with PCS is causal or correlated. Furthermore, depression and anxiety might be risk factors for initially developing PCS. ${ }^{71,72}$ Yet, studies on rodents have found evidence of causality, in which induced mTBI disrupts limbic system function, subsequently leading to long lasting depressive, fearful and anxious behavior. ${ }^{73-75}$ Whatever the case, the neuropsychological nature of PCS makes providing psychosocial coping strategies a viable part of the multidisciplinary approach, and some reviews have supported the use of cognitive behavioral therapy in improving psychosocial outcomes ${ }^{76}$; however, more research is needed.

Meditation and mindfulness, ${ }^{77-80}$ body awareness therapy ${ }^{81-83}$ breathing exercises and stretching, ${ }^{84,85}$ have shown to be effective ways of modulating the ANS, lower sympathetic activity and improve biomarkers of stress in various populations. Little evidence exists on treatments specifically addressing dysautonomia as a result of mTBI, although one pilot study did show improvements in QOL and self-efficacy on a group of PCS patients after undergoing a mindfulness-based intervention. ${ }^{86}$ Moreover, studies show that meditation can improve cognition and regulate $\mathrm{CBF},{ }^{77,87}$ making it an area of interest considering that $\mathrm{CBF}$ disruptions are linked to mTBI and PCS symptomatology. ${ }^{24,88}$ The efficacy of meditation techniques and body awareness strategies can vary between individuals ${ }^{89}$ and some techniques can be harder to conceptualize than others, 
which was illustrated by Case 3 who expressed difficulty in this regard. Our modified BBAT intervention in Study B was directed at addressing the high arousal and stimuli intolerant state of our cases, hopefully modulating the ANS and reducing PCS symptoms. While both cases reported positive associations with the sessions, only Case 4 experienced symptom relief and significant improved QOL. Considering that the intervention lasted 3 weeks, a longer duration of exposure may have been required to more accurately assess the protocol, as a duration of up to 8 weeks has been necessary before experiencing the full benefits of similar methods. ${ }^{90}$

Exercise intolerance is a common phenomenon in PCS patients and all of our cases reported having experienced symptom exacerbation during physical activity. Studies have found increased blood pressure (BP), $\mathrm{HR}$ and arterial CO2 in PCS patients compared to healthy controls during exercise, which may be linked to the changes in CBF and a reduced ability of the ANS to regulate orthostatic BP. ${ }^{22,91,92}$ This exercise intolerance occurs at different ranges of $\mathrm{HR}$ and while finding the appropriate range for the individual can be challenging, abstaining from exercise and physical activity entirely is not recommended. ${ }^{92}$ Both cases in Study A showed a significant decrease in their PCS symptoms post-intervention and were ultimately able to increase their HR intensity, relative to their maximum HR, during exercise by $15 \%$ and $12 \%$, for Case 1 and 2 respectively, without symptom exacerbation. However, as the cases simultaneously underwent cervical treatment, it is not possible to isolate the results of the exercise intervention completely. Yet our findings are in line with other studies showing improvements in physiological exercise biomarkers and PCS symptoms using similar protocols. ${ }^{59,91,93}$ This may be partly due to changes in CBF, as a pilot study, using fMRI, found that PCS patients had a normalization of brain activity and CBF after the implementation of a submaximal aerobic intervention. ${ }^{94}$ An important aspect of the protocol is keeping intensity levels below symptom threshold, before gradually increasing the intensity while assessing individual response. Early initiation of aerobic exercise has been associated with a faster recovery ${ }^{95}$; however, overexertion at different stages post-mTBI can be detrimental ${ }^{15,96-98}$ and some evidence suggests that the appropriate intensity is timing-based.$^{99}$ It is therefore recommended that practitioners monitor progress under controlled conditions and with the proper testing tools. A high quality and specifically developed method for testing this is the Buffalo Concussion Treadmill Test, ${ }^{100}$ but other testing methods with comparable protocols could be similarly applied.

Whiplash is commonly associated with PCS and exists both as part of the symptomatology, as well as a comorbidity. It has similar injury biomechanics to mTBI, such as the abrupt acceleration and deceleration of the cervical spine and head, and shares a majority of symptoms. ${ }^{101}$ One of the underlying mechanisms behind this may be cranial nerve trauma, including the vagus nerve which regulates the ANS, ${ }^{102,103}$ either as a direct result of impact, or as a secondary effect based on changes in arthrokinematics and joint degeneration. ${ }^{104,105}$ Cervical trauma has been linked to oculomotor deficiencies ${ }^{32,106,107}$ and whether as a result of whiplash or otherwise, issues with convergence, accommodation and saccades occur regularly after mTBI and often lingers in patients with PCS. ${ }^{19,108}$ Screening for this, and possibly referring to neuro-ophthalmologic treatment, is recommended. ${ }^{109}$

All our cases reported cervical stiffness and pain, and evidence shows that a high prevalence of various cervical and periarticular impairments are common after a mTBI. These include postural imbalances, myofascial triggers, muscle weakness, decreased joint mobility and weakness of cervical flexors associated with cervicogenic headaches. ${ }^{110,111}$ Considering this, and that up to $50 \%$ of whiplash patients still report symptoms a year after injury, ${ }^{112}$ cervical pathology is a critical consideration in the PCS rehabilitation. ${ }^{113-115}$ Both cases in Study A reported reductions in cervical associated symptoms such as dizziness, nausea, and headaches, although only Case 2 improved in CCFT grading. These findings are inconclusive, but other studies have suggested that deep flexor strengthening can have a positive effect on neck-related issues in some PCS patients ${ }^{114}$ and that cervical and vestibular rehabilitation may reduce cervicogenic symptoms. ${ }^{116,117} \mathrm{~A}$ variety of manual treatments may also improve outcomes, however, efficacy seems highly individual and more research is needed. ${ }^{113,118}$ Clinically, some PCS patients are sensitive to cervical treatment and may respond with symptom exacerbation to mobilizations, manipulations, strengthening exercises and even stretching. 


\subsection{Clinical Expertise}

When initiating the PCS rehabilitation, identifying potential physical and mental barriers to activity, and acknowledging these limitations, is an important part of the planning. The process requires submaximal testing and treatment, and a deliberate slow progression as symptom thresholds are often low in the early stages. By taking inspiration from other chronic pain protocols, a proper pacing strategy can be useful in building tolerance to the treatment, ${ }^{119}$ and using positive reinforcement and education on the nature of PCS can help demystify the condition. This includes focusing on solutions and brain plasticity instead of limitations when communicating with the patient, after the initial explanation on pathology, and may help avoid the unwanted nocebo effects that many chronic pain patients can be susceptible to. ${ }^{120,121}$ Setbacks and symptom volatility are to be expected even with thoughtful consideration to programming, which is why the practitioner benefits from listening to the patient and adjusting volume and intensity accordingly, as well as including an appropriate frequency of examination and re-testing. Working on acceptance of current disability may help improve emotional outcomes and patient self-efficacy, ${ }^{122}$ and celebrating short term goals helps improve patient motivation and long term compliance. ${ }^{123,124}$ Lastly, a key part of administering the rehabilitation is knowing when a referral is indicated and if treatment is outside the bounds of expertise of the practitioner.

\subsection{Patient preferences}

PCS is in many ways a "hidden condition" not easily visible and with no current standardized biomarkers for diagnosis, ${ }^{125}$ and the path towards rehabilitation is often confusing, and uncertain, for patients and practitioners alike. Most general practitioners are not well equipped to treat PCS patients, which is highlighted by the lack of standardized care, and rest is often the only offered treatment strategy. Our cases expressed dissatisfaction with their healthcare providers and felt neglected, as well as not having been taken seriously. One of the emerging themes derived from the cases was the importance of meeting other patients in similar circumstances who could relate to them and inhabit an acknowledging space to freely share experiences and discuss daily obstacles. This acknowledgement and social exchange was seen as a central part of coping with the condition, and should be viewed by practitioners as a legitimate consideration for rehabilitation in the clinical practice. The use of deliberate framing and the facilitation of a constructive and accommodating environment in our studies were beneficial in dealing with the psychosocial challenges. Cases further highlighted that being in a safe environment, and adjusting for unwanted mental and physical overstimulation, resulted in less exacerbation of symptoms. Besides the benefits of rehabilitation on their mood, stamina and cognitive function, our cases expressed a sense of meaning in their daily lives when having something to attend, especially considering that three of the cases were on sick leave, with none regularly engaging in scheduled social events. The interventions themselves were seen as motivating factors, regardless of any improvement in symptoms, suggesting that an active approach has an inherent advantage compared to a passive or non-active approach even before considering outcomes. Through the gathering of the qualitative data, including the group feedback sessions, our cases helped clarify valuable areas of concern illuminating patient preferences and individual perspectives on the intervention protocols which would not have been possible otherwise.

\subsection{Model of Conclusion}

By taking advantage of the three dimensions of the EBP model as a methodological approach to the PCS cases, this paper incorporated the: Best Research Evidence, Clinical Expertise and Patient Preferences, through an examination of the benefits of two distinct intervention protocols. These results illustrate how an active and individualized approach can represent important qualities that can be applied into further and larger studies, and we recommend additional studies regarding exposure to active, individualized and graded treatments to patients dealing with PCS. Based on the results and discussion in this paper, we have summarized relevant findings and suggestions in the following modified EBP model (Fig. 7), which may be of help to practitioners in the clinical practice. 


\section{FIGURE 7}

\section{References}

1. Pinner M, Børgensen SE, Jensen R, Birket-Smith M, Gade A, Jo. R.Konsensusrapport Om Commotio Cerebri (hjernerystelse). In: Consensus-Driven Guidelines Regarding Commotio Cerebri . Skouby, Denmark: Videnscenter for Hjerneskade;2003:9.

2. Rytter HM, Mogensen J. Commotio cerebri og mild traumatisk hjerneskade - er vi enige om, hvad det er? BestPractice Psykiatri/Neurologi . 2017;36:30-33.

3. Cassidy JD, Carroll LJ, Peloso PM, et al. Incidence, risk factors and prevention of mild traumatic brain injury: results of the WHO Collaborating Centre Task Force on Mild Traumatic Brain Injury. J Rehabil Med . 2004;(43 Suppl):28-60. doi:10.1080/16501960410023732

4. Polinder S, Cnossen MC, Real RGL, et al. A Multidimensional Approach to Post-concussion Symptoms in Mild Traumatic Brain Injury. Front Neurol . 2018;9. doi:10.3389/fneur.2018.01113

5. Hiploylee C, Dufort PA, Davis HS, et al. Longitudinal Study of Postconcussion Syndrome: Not Everyone Recovers. J Neurotrauma . 2017;34(8):1511. doi:10.1089/neu.2016.4677

6. Deleu N, Christiansen P, Henriksen H. Patienter med hjernerystelse.Neurologi . 2017:719-727.

7. American Psychiatric Association. Diagnostic and Statistical Manual of Mental Disorders (DSM-5ß) . American Psychiatric Pub; 2013.

8. Rasmussen MM, Clemmensen D, Jensen SS. [Prolonged Symptoms Following Mild Traumatic Brain Injury]. Ugeskr Laeger . 2010;172(39). https://pubmed.ncbi.nlm.nih.gov/20920395/. Accessed June 5, 2020.

9. Guillamondegui OD, Montgomery SA, Phibbs FT, et al. Traumatic Brain Injury and Depression . Rockville, MD 20850: Agency for Healthcare Research and Quality; 2011. https://effectivehealthcare.ahrq.gov/sites/default/files/pdf/depression-brain-injury_research.pdf. Accessed May 28, 2020.

10. Leddy JJ, Wilber CG, Willer BS. Active Recovery From Concussion.Curr Opin Neurol . 2018;31(6). doi:10.1097/WCO.0000000000000611

11. Silverberg ND, Iverson GL. Is Rest After Concussion "The Best Medicine?": Recommendations for Activity Resumption Following Concussion in Athletes, Civilians, and Military Service Members. J Head Trauma Rehabil . 2013;28(4). doi:10.1097/HTR.0b013e31825ad658

12. Lempke L, Jaffri A, Erdman N. The Effects of Early Physical Activity Compared to Early Physical Rest on Concussion Symptoms. J Sport Rehabil . 2019;28(1):99-105. doi:10.1123/jsr.2017-0217

13. McCrory P, Meeuwisse W, Dvorak J, et al. Consensus statement on concussion in sport - the 5th international conference on concussion in sport held in Berlin, October 2016. Br J Sports Med . 2017;51(11):838-847. doi:10.1136/bjsports-2017-097699

14. Brown NJ, Mannix RC, O'Brien MJ, et al. Effect of Cognitive Activity Level on Duration of PostConcussion Symptoms. Pediatrics . 2014;133(2):e299. doi:10.1542/peds.2013-2125

15. Sawyer Q, Vesci B, Valovich McLeod TC. Physical Activity and Intermittent Postconcussion Symptoms After a Period of Symptom-Limited Physical and Cognitive Rest. J Athl Train . 2016;51(9):739. doi:10.4085/1062-6050-51.12.01

16. Schneider KJ, Leddy JJ, Guskiewicz KM, et al. Rest and treatment/rehabilitation following sport-related concussion: a systematic review. Br J Sports Med . 2017;51(12):930-934. doi:10.1136/bjsports-2016-097475

17. Broglio SP, Collins MW, Williams RM, Mucha A, Kontos A. Current and emerging rehabilitation for concussion: A review of the evidence.Clin Sports Med . 2015;34(2):213. doi:10.1016/j.csm.2014.12.005 
18. Postconcussion syndrome. In: Handbook of Clinical Neurology . Vol 158. Elsevier; 2018:163-178. doi:10.1016/B978-0-444-63954-7.00017-3

19. Heitger MH, Jones RD, Macleod AD, Snell DL, Frampton CM, Anderson TJ. Impaired eye movements in post-concussion syndrome indicate suboptimal brain function beyond the influence of depression, malingering or intellectual ability. Brain . 2009;132(10):2850-2870. doi:10.1093/brain/awp181

20. Clark J, Hasselfeld K, Bigsby K, Divine J. Colored Glasses to Mitigate Photophobia Symptoms Posttraumatic Brain Injury. J Athl Train . 2017;52(8):725. doi:10.4085/1062-6050-52.4.04

21. Assi H, Davis Moore R, Ellemberg D, Hébert S. Sensitivity to sounds in sport-related concussed athletes: a new clinical presentation of hyperacusis. Sci Rep . 2018;8(1):1-8. doi:10.1038/s41598-018-28312-1

22. Kozlowski KF, Graham J, Leddy JJ, Devinney-Boymel L, Willer BS. Exercise Intolerance in Individuals With Postconcussion Syndrome.J Athl Train . 2013;48(5). doi:10.4085/1062-6050-48.5.02

23. Meier TB, Bellgowan PS, Singh R, Kuplicki R, Polanski DW, Mayer AR. Recovery of Cerebral Blood Flow Following Sports-Related Concussion.JAMA Neurol . 2015;72(5). doi:10.1001/jamaneurol.2014.4778

24. Stephens JA, Liu P, Lu H, Suskauer SJ. Cerebral Blood Flow After Mild Traumatic Brain Injury: Associations Between Symptoms and Post-Injury Perfusion. J Neurotrauma . 2018;35(2). doi:10.1089/neu.2017.5237

25. Giza CC, Hovda DA. The New Neurometabolic Cascade of Concussion. Neurosurgery . 2014;75 Suppl 4(0 4). doi:10.1227/NEU.0000000000000505

26. Johnson VE, Stewart W, Smith DH. Axonal Pathology in Traumatic Brain Injury. Exp Neurol . 2013;246. doi:10.1016/j.expneurol.2012.01.013

27. Pertab JL, Merkley TL, Cramond AJ, Cramond K, Paxton H, Wu T. Concussion and the Autonomic Nervous System: An Introduction to the Field and the Results of a Systematic Review.NeuroRehabilitation . 2018;42(4). doi:10.3233/NRE-172298

28. Dmitry Esterov BDG. Autonomic Dysfunction after Mild Traumatic Brain Injury. Brain Sciences . 2017;7(8). doi:10.3390/brainsci7080100

29. Undurti A, Colasurdo EA, Sikkema CL, et al. Chronic Hypopituitarism Associated With Increased Postconcussive Symptoms Is Prevalent After Blast-Induced Mild Traumatic Brain Injury. Front Neurol . 2018;9. doi:10.3389/fneur.2018.00072

30. Di Battista AP, Rhind SG, Churchill N, Richards D, Lawrence DW, Hutchison MG. Peripheral blood neuroendocrine hormones are associated with clinical indices of sport-related concussion. Sci Rep . 2019;9(1):110. doi:10.1038/s41598-019-54923-3

31. Treleaven J, Jull G, Atkinson L. Cervical Musculoskeletal Dysfunction in Post-Concussional Headache. Cephalalgia . 1994;14(4). doi:10.1046/j.1468-2982.1994.1404273.x

32. Barnett BP, Singman EL. Vision Concerns After Mild Traumatic Brain Injury. Curr Treat Options Neurol . 2015;17(2). doi:10.1007/s11940-014-0329-y

33. Ognyan I. Kolev MS. Vestibular disorders following different types of head and neck trauma. Funct Neurol . 2016;31(2):75. doi:10.11138/FNeur/2016.31.2.075

34. Prince C, Bruhns ME. Evaluation and Treatment of Mild Traumatic Brain Injury: The Role of Neuropsychology. Brain sciences . 2017;7(8). doi:10.3390/brainsci7080105

35. Cole WR, Bailie JM. Neurocognitive and Psychiatric Symptoms Following Mild Traumatic Brain Injury. https://pubmed.ncbi.nlm.nih.gov/26583174/. Accessed May 28, 2020.

36. Guskiewicz KM, Register-Mihalik JK. Postconcussive Impairment Differences Across a Multifaceted Concussion Assessment Protocol.PM R . 2011;3(10 Suppl 2). doi:10.1016/j.pmrj.2011.08.009 
37. Sackett DL, Rosenberg WMC, Muir Gray JA, Brian Haynes R, Scott Richardson W. Evidence based medicine: what it is and what it isn't.BMJ . 1996;312(7023):71-72. doi:10.1136/bmj.312.7023.71

38. Straus SE, Richardson WS, Glasziou P, Haynes RB.Evidence-Based Medicine: How to Practice and Teach EBM (3rd Ed.) . Edinburgh, UK: Elsevier Churchill Livingstone; 2005.

39. Howick J. The Philosophy of Evidence-Based Medicine. 2011. doi:10.1002/9781444342673

40. Malterud K. Kvalitative metoder i medisinsk forskning — en innføring, 2.utg. Nordic Journal of Nursing Research . 2003;23(2):50-50. doi:10.1177/010740830302300212

41. Pedersen M, Bundgaard TH, Zeeman P, et al. Action Research in Rehabilitation With Chronic Stroke Recovery: A Case Report With a Focus on Neural Plasticity. NeuroRehabilitation . 2016;39(2). doi:10.3233/NRE-161356

42. WMA - The World Medical Association-WMA Declaration of Helsinki - Ethical Principles for Medical Research Involving Human Subjects. https://www.wma.net/policies-post/wma-declaration-of-helsinkiethical-principles-for-medical-research-involving-human-subjects/. Accessed May 28, 2020.

43. Cieza A, Stucki G. The International Classification of Functioning Disability and Health: Its Development Process and Content Validity.Eur J Phys Rehabil Med . 2008;44(3). https://pubmed.ncbi.nlm.nih.gov/18762740/. Accessed June 5, 2020.

44. King NS, Crawford S, Wenden FJ, Moss NE, Wade DT. The Rivermead Post Concussion Symptoms Questionnaire: A Measure of Symptoms Commonly Experienced After Head Injury and Its Reliability. $J$ Neurol . 1995;242(9). doi:10.1007/BF00868811

45. Jull GA, O'Leary SP, Falla DL. Clinical Assessment of the Deep Cervical Flexor Muscles: The Craniocervical Flexion Test. J Manipulative Physiol Ther . 2008;31(7). doi:10.1016/j.jmpt.2008.08.003

46. Ware JE, Sherbourne CD. The MOS 36-item Short-Form Health Survey (SF-36). I. Conceptual Framework and Item Selection. Med Care . 1992;30(6). https://pubmed.ncbi.nlm.nih.gov/1593914/. Accessed June 5,2020 .

47. Eyres S, Carey A, Gilworth G, Neumann V, Tennant A. Construct Validity and Reliability of the Rivermead Post-Concussion Symptoms Questionnaire. Clin Rehabil . 2005;19(8). doi:10.1191/0269215505cr905oa

48. Jørgensen R, Ris I, Falla D, Juul-Kristensen B. Reliability, construct and discriminative validity of clinical testing in subjects with and without chronic neck pain. BMC Musculoskelet Disord . 2014;15. doi:10.1186/1471-2474-15-408

49. Simon NM, Otto MW, Korbly NB, Peters PM, Nicolaou DC, Pollack MH. Quality of Life in Social Anxiety Disorder Compared With Panic Disorder and the General Population. Psychiatr Serv . 2002;53(6). doi:10.1176/appi.ps.53.6.714

50. Shu-Wen SU DW. The Reliability and Validity of Short Form-12 Health Survey Version 2 for Chinese Older Adults. Iran J Public Health . 2019;48(6):1014. https://www.ncbi.nlm.nih.gov/pmc/articles/PMC6635330/. Accessed May 28, 2020.

51. Maruish ME. User's Manual for the SF-36v2 Health Survey 3rd Edition . Quality Metric Incorporated; 2011.

52. Short Form 36 (SF-36). https://fysio.dk/fafo/maleredskaber/short-form-36-sf-36. Accessed May 28, 2020.

53. Brinkmann S, Kvale S. InterViews: Learning the Craft of Qualitative Research Interviewing . SAGE Publications, Incorporated; 2014.

54. Panjabi MM. The Stabilizing System of the Spine. Part II. Neutral Zone and Instability Hypothesis. $J$ Spinal Disord . 1992;5(4). doi:10.1097/00002517-199212000-00002 
55. Roxendal G, Winberg A. Levande människa: basal kroppskännedom för rörelse och vila . Natur och Kultur; 2002.

56. Skjaerven LH, Mattsson M, Catalan-Matamoros D, Parker A, Gard G, Gyllensten AL. Consensus on Core Phenomena and Statements Describing Basic Body Awareness Therapy Within the Movement Awareness Domain in Physiotherapy. Physiother Theory Pract . 2019;35(1). doi:10.1080/09593985.2018.1434578

57. Ellis MJ, Leddy JJ, Willer B. Physiological, Vestibulo-Ocular and Cervicogenic Post-Concussion Disorders: An Evidence-Based Classification System With Directions for Treatment. Brain Inj . 2015;29(2). doi:10.3109/02699052.2014.965207

58. Sandel N, Reynolds E, Cohen PE, Gillie BL, Kontos AP. Anxiety and Mood Clinical Profile following Sport-related Concussion: From Risk Factors to Treatment. Sport, exercise, and performance psychology . 2017;6(3):304. doi:10.1037/spy0000098

59. Leddy J, Baker JG, Haider MN, Hinds A, Willer B. A Physiological Approach to Prolonged Recovery From Sport-Related Concussion. J Athl Train . 2017;52(3):299. doi:10.4085/1062-6050-51.11.08

60. Maruta J, Lumba-Brown A, Ghajar J. Concussion Subtype Identification With the Rivermead Postconcussion Symptoms Questionnaire. Front Neurol . 2018;9. doi:10.3389/fneur.2018.01034

61. Harrison AT, Gunn BS, Kay JJM, Moore RD. The influence of concussion on cardio-autonomic function during cognition before and after exercise.Neurology . 2018;91(23 Supplement 1):S9-S9. doi:10.1212/01.wnl.0000550663.80652.c4

62. Blake TA, McKay CD, Meeuwisse WH, Emery CA. The Impact of Concussion on Cardiac Autonomic Function: A Systematic Review. Brain Inj . 2016;30(2). doi:10.3109/02699052.2015.1093659

63. Kreibig SD. Autonomic Nervous System Activity in Emotion: A Review.Biol Psychol . 2010;84(3). doi:10.1016/j.biopsycho.2010.03.010

64. Friedman BH. An autonomic flexibility-neurovisceral integration model of anxiety and cardiac vagal tone. Biol Psychol . 2007;74(2):185-199. doi:10.1016/j.biopsycho.2005.08.009

65. Lindquist KA, Wager TD, Kober H, Bliss-Moreau E, Barrett LF. The brain basis of emotion: A metaanalytic review. Behav Brain Sci . 2012;35(3):121. doi:10.1017/S0140525X11000446

66. Arciniegas DB, Alan Anderson C, Topkoff J, McAllister TW. Mild traumatic brain injury: a neuropsychiatric approach to diagnosis, evaluation, and treatment. Neuropsychiatr Dis Treat . 2005;1(4):311. https://www.ncbi.nlm.nih.gov/pmc/articles/PMC2424119/. Accessed May 28, 2020.

67. Moore EL, Terryberry-Spohr L, Hope DA. Mild Traumatic Brain Injury and Anxiety Sequelae: A Review of the Literature. Brain Inj . 2006;20(2). doi:10.1080/02699050500443558

68. Vargas G, Rabinowitz A, Meyer J, Arnett PA. Predictors and Prevalence of Postconcussion Depression Symptoms in Collegiate Athletes.J Athl Train . 2015;50(3):250. doi:10.4085/1062-6050-50.3.02

69. Louise C. Hawkley JPC. Perceived social isolation, evolutionary fitness and health outcomes: a lifespan approach. Philos Trans R Soc Lond B Biol Sci . 2015;370(1669). doi:10.1098/rstb.2014.0114

70. Ge L, Yap CW, Ong R, Heng BH. Social isolation, loneliness and their relationships with depressive symptoms: A population-based study.PLoS One . 2017;12(8):e0182145. doi:10.1371/journal.pone.0182145

71. Yue JK, Cnossen MC, Winkler EA, et al. Pre-injury Comorbidities Are Associated With Functional Impairment and Post-concussive Symptoms at 3- And 6-Months After Mild Traumatic Brain Injury: A TRACK-TBI Study.Front Neurol . 2019;10. doi:10.3389/fneur.2019.00343

72. Oldenburg C, Lundin A, Edman G, Deboussard CN, Bartfai A. Emotional reserve and prolonged postconcussive symptoms and disability: a Swedish prospective 1-year mild traumatic brain injury cohort study. BMJ Open . 2018;8(7):e020884. doi:10.1136/bmjopen-2017-020884 
73. Almeida-Suhett CP, Prager EM, Pidoplichko V, et al. Reduced GABAergic Inhibition in the Basolateral Amygdala and the Development of Anxiety-Like Behaviors after Mild Traumatic Brain Injury. PLoS One . 2014;9(7):e102627. doi:10.1371/journal.pone.0102627

74. Meyer DL, Davies DR, Barr JL, Manzerra P, Forster GL. Mild Traumatic Brain Injury in the Rat Alters Neuronal Number in the Limbic System and Increases Conditioned Fear and Anxiety-Like Behaviors. Exp Neurol . 2012;235(2). doi:10.1016/j.expneurol.2012.03.012

75. Bajwa NM, Halavi S, Hamer M, et al. Mild Concussion, but Not Moderate Traumatic Brain Injury, Is Associated with Long-Term Depression-Like Phenotype in Mice. PLoS One . 2016;11(1):e0146886. doi:10.1371/journal.pone.0146886

76. Al Sayegh A, Sandford D, Carson AJ. Psychological approaches to treatment of postconcussion syndrome: a systematic review. J Neurol Neurosurg Psychiatry . 2010;81(10):1128-1134. doi:10.1136/jnnp.2008.170092

77. Newberg AB, Wintering N, Khalsa DS, Roggenkamp H, Waldman MR. Meditation Effects on Cognitive Function and Cerebral Blood Flow in Subjects With Memory Loss: A Preliminary Study. J Alzheimers Dis . 2010;20(2). doi:10.3233/JAD-2010-1391

78. Parmentier FBR, García-Toro M, García-Campayo J, Yañez AM, Andrés P, Gili M. Mindfulness and Symptoms of Depression and Anxiety in the General Population: The Mediating Roles of Worry, Rumination, Reappraisal and Suppression. Front Psychol . 2019;10. doi:10.3389/fpsyg.2019.00506

79. Tang YY, Hölzel BK, Posner MI. The Neuroscience of Mindfulness Meditation. Nat Rev Neurosci . 2015;16(4). doi:10.1038/nrn3916

80. Pascoe MC, Thompson DR, Jenkins ZM, Ski CF. Mindfulness Mediates the Physiological Markers of Stress: Systematic Review and Meta-Analysis.J Psychiatr Res . 2017;95. doi:10.1016/j.jpsychires.2017.08.004

81. Mantovani AM, Fregonesi CE, Lorençoni RM, et al. Immediate Effect of Basic Body Awareness Therapy on Heart Rate Variability. Complement Ther Clin Pract . 2016;22. doi:10.1016/j.ctcp.2015.10.003

82. Van der Maas LC, Köke A, Bosscher RJ, Twisk JW, Janssen TW, Peters M. Body Awareness as an Important Target in Multidisciplinary Chronic Pain Treatment: Mediation and Subgroup Analyses. Clin J Pain . 2016;32(9). doi:10.1097/AJP.0000000000000320

83. Blaauwendraat C, A LB, Gyllensten AL. One-year Follow-Up of Basic Body Awareness Therapy in Patients With Posttraumatic Stress Disorder. A Small Intervention Study of Effects on Movement Quality, PTSD Symptoms, and Movement Experiences. Physiother Theory Pract . 2017;33(7). doi:10.1080/09593985.2017.1325957

84. Zaccaro A, Piarulli A, Laurino M, et al. How Breath-Control Can Change Your Life: A Systematic Review on Psycho-Physiological Correlates of Slow Breathing. Front Hum Neurosci . 2018;12. doi:10.3389/fnhum.2018.00353

85. Farinatti PT, Brandão C, Soares PP, Duarte AF. Acute Effects of Stretching Exercise on the Heart Rate Variability in Subjects With Low Flexibility Levels. J Strength Cond Res . 2011;25(6). doi:10.1519/JSC.0b013e3181e06ce1

86. Azulay J, Smart CM, Mott T, Cicerone KD. A Pilot Study Examining the Effect of Mindfulness-Based Stress Reduction on Symptoms of Chronic Mild Traumatic Brain Injury/Postconcussive Syndrome. J Head Trauma Rehabil . 2013;28(4). doi:10.1097/HTR.0b013e318250ebda

87. Last N, Tufts E, Auger LE. The Effects of Meditation on Grey Matter Atrophy and Neurodegeneration: A Systematic Review. J Alzheimers Dis . 2017;56(1). doi:10.3233/JAD-160899

88. Ellis MJ, Ryner LN, Sobczyk O, et al. Neuroimaging Assessment of Cerebrovascular Reactivity in Concussion: Current Concepts, Methodological Considerations, and Review of the Literature. Front Neurol . 2016;7. doi:10.3389/fneur.2016.00061 
89. Steinhubl SR, Wineinger NE, Patel S, et al. Cardiovascular and nervous system changes during meditation. Front Hum Neurosci . 2015;9. doi:10.3389/fnhum.2015.00145

90. Basso JC, McHale A, Ende V, Oberlin DJ, Suzuki WA. Brief, Daily Meditation Enhances Attention, Memory, Mood, and Emotional Regulation in Non-Experienced Meditators. Behav Brain Res . 2019;356. doi:10.1016/j.bbr.2018.08.023

91. Leddy JJ, Haider MN, Ellis M, Willer BS. Exercise is Medicine for Concussion. Curr Sports Med Rep . 2018;17(8):262. doi:10.1249/JSR.0000000000000505

92. Leddy JJ, Kozlowski K, Fung M, Pendergast DR, Willer B. Regulatory and Autoregulatory Physiological Dysfunction as a Primary Characteristic of Post Concussion Syndrome: Implications for Treatment.NeuroRehabilitation . 2007;22(3). https://pubmed.ncbi.nlm.nih.gov/17917170/. Accessed May 28, 2020 .

93. Baker JG, Freitas MS, Leddy JJ, Kozlowski KF, Willer BS. Return to Full Functioning After Graded Exercise Assessment and Progressive Exercise Treatment of Postconcussion Syndrome. Rehabil Res Pract . 2012;2012. doi:10.1155/2012/705309

94. Leddy JJ, Cox JL, Baker JG, et al. Exercise Treatment for Postconcussion Syndrome: A Pilot Study of Changes in Functional Magnetic Resonance Imaging Activation, Physiology, and Symptoms. J Head Trauma Rehabil . 2013;28(4). doi:10.1097/HTR.0b013e31826da964

95. Lawrence DW, Richards D, Comper P, Hutchison MG. Earlier time to aerobic exercise is associated with faster recovery following acute sport concussion. PLoS One . 2018;13(4):e0196062. doi:10.1371/journal.pone.0196062

96. Majerske CW, Mihalik JP, Ren D, et al. Concussion in Sports: Postconcussive Activity Levels, Symptoms, and Neurocognitive Performance. J Athl Train . 2008;43(3):265. doi:10.4085/1062-6050-43.3.265

97. Imhoff S, Malenfant S, Nadreau É, Poirier P, Bailey DM, Brassard P. Uncoupling between cerebral perfusion and oxygenation during incremental exercise in an athlete with postconcussion syndrome: a case report.Physiological Reports . 2017;5(2). doi:10.14814/phy2.13131

98. Lishchynsky JT, Rutschmann TD, Toomey CM, et al. The Association Between Moderate and Vigorous Physical Activity and Time to Medical Clearance to Return to Play Following Sport-Related Concussion in Youth Ice Hockey Players. Front Neurol . 2019;10. doi:10.3389/fneur.2019.00588

99. Remigio-Baker RA, Bailie JM, Gregory E, et al. Activity Level and Type During Post-acute Stages of Concussion May Play an Important Role in Improving Symptoms Among an Active Duty Military Population.Front Neurol . 2019;10. doi:10.3389/fneur.2019.00602

100. Haider MN, Leddy JJ, Wilber CG, et al. The Predictive Capacity of the Buffalo Concussion Treadmill Test After Sport-Related Concussion in Adolescents. Front Neurol . 2019;10. doi:10.3389/fneur.2019.00395

101. Evans RW. Persistent Post-Traumatic Headache, Postconcussion Syndrome, and Whiplash Injuries: The Evidence for a Non-Traumatic Basis With an Historical Review. Headache . 2010;50(4). doi:10.1111/j.15264610.2010.01645.x

102. Deuchars SA, Lall VK, Clancy J, et al. Mechanisms underpinning sympathetic nervous activity and its modulation using transcutaneous vagus nerve stimulation. Exp Physiol . 2018;103(3):326. doi:10.1113/EP086433

103. Ernsberger U, Rohrer H. Sympathetic tales: subdivisons of the autonomic nervous system and the impact of developmental studies. Neural Dev . 2018;13(1):1-21. doi:10.1186/s13064-018-0117-6

104. Coello AF, Canals AG, Gonzalez JM, Martín JJ. Cranial Nerve Injury After Minor Head Trauma. $J$ Neurosurg . 2010;113(3). doi:10.3171/2010.6.JNS091620 
105. Matuszak JM, McVige J, McPherson J, Willer B, Leddy J. A Practical Concussion Physical Examination Toolbox: Evidence-Based Physical Examination for Concussion. Sports Health . 2016;8(3):260. doi:10.1177/1941738116641394

106. Zaremski JL, Herman DC, Clugston JR, Hurley RW, Ahn AH. Occipital Neuralgia as a Sequela of Sports Concussion: A Case Series and Review of the Literature. Curr Sports Med Rep . 2015;14(1):16. doi:10.1249/JSR.0000000000000121

107. Treleaven J. Sensorimotor Disturbances in Neck Disorders Affecting Postural Stability, Head and Eye Movement Control. Man Ther . 2008;13(1). doi:10.1016/j.math.2007.06.003

108. Debacker J, Ventura R, Galetta SL, Balcer LJ, Rucker JC. Neuro-ophthalmologic Disorders Following Concussion. Handb Clin Neurol . 2018;158. doi:10.1016/B978-0-444-63954-7.00015-X

109. Gallaway M, Scheiman M, Mitchell GL. Vision Therapy for Post-Concussion Vision Disorders. Optom Vis Sci . 2017;94(1). doi:10.1097/OPX.0000000000000935

110. Tiwari D, Goldberg A, Yorke A, Marchetti GF, Alsalaheen B. CHARACTERIZATION OF CERVICAL SPINE IMPAIRMENTS IN CHILDREN AND ADOLESCENTS POST-CONCUSSION. Int $J$ Sports Phys Ther . 2019;14(2):282. https://www.ncbi.nlm.nih.gov/pmc/articles/PMC6449018/. Accessed May 28, 2020.

111. Watson DH, Trott PH. Cervical Headache: An Investigation of Natural Head Posture and Upper Cervical Flexor Muscle Performance.Cephalalgia . 1993;13(4). doi:10.1046/j.1468-2982.1993.1304272.x

112. Carroll LJ, Holm LW, Hogg-Johnson S, et al. Course and Prognostic Factors for Neck Pain in WhiplashAssociated Disorders (WAD): Results of the Bone and Joint Decade 2000-2010 Task Force on Neck Pain and Its Associated Disorders. Eur Spine J . 2008;17(Suppl 1):83. doi:10.1007/s00586-008-0628-7

113. Morin M, Langevin P, Fait P. Cervical Spine Involvement in Mild Traumatic Brain Injury: A Review. J Sports Med . 2016;2016. doi:10.1155/2016/1590161

114. Marshall CM, Vernon H, Leddy JJ, Baldwin BA. The Role of the Cervical Spine in Post-Concussion Syndrome. Phys Sportsmed . 2015;43(3). doi:10.1080/00913847.2015.1064301

115. Kennedy E, Quinn D, Chapple C, Tumilty S. Can the Neck Contribute to Persistent Symptoms Post Concussion? A Prospective Descriptive Case Series. J Orthop Sports Phys Ther . 2019;49(11). doi:10.2519/jospt.2019.8547

116. Schneider KJ, Meeuwisse WH, Nettel-Aguirre A, et al. Cervicovestibular Rehabilitation in Sport-Related Concussion: A Randomised Controlled Trial. Br J Sports Med . 2014;48(17). doi:10.1136/bjsports-2013093267

117. Alsalaheen BA, Mucha A, Morris LO, et al. Vestibular Rehabilitation for Dizziness and Balance Disorders After Concussion. J Neurol Phys Ther . 2010;34(2). doi:10.1097/NPT.0b013e3181dde568

118. Morikawa Y, Takamoto K, Nishimaru H, et al. Compression at Myofascial Trigger Point on Chronic Neck Pain Provides Pain Relief through the Prefrontal Cortex and Autonomic Nervous System: A Pilot Study. Front Neurosci . 2017;11. doi:10.3389/fnins.2017.00186

119. Antcliff D, Keenan AM, Keeley P, Woby S, McGowan L. Survey of Activity Pacing Across Healthcare Professionals Informs a New Activity Pacing Framework for Chronic Pain/Fatigue. Musculoskeletal Care . 2019;17(4). doi:10.1002/msc.1421

120. Klinger R, Blasini M, Schmitz J, Colloca L. Nocebo effects in clinical studies: hints for pain therapy. Pain Reports . 2017;2(2). doi:10.1097/PR9.0000000000000586

121. Colloca L. The Placebo Effect in Pain Therapies. Annu Rev Pharmacol Toxicol . 2019;59:191. doi:10.1146/annurev-pharmtox-010818-021542 
122. Yehene E, Lichtenstern G, Harel Y, Druckman E, Sacher Y. Self-efficacy and Acceptance of Disability Following Mild Traumatic Brain Injury: A Pilot Study. Appl Neuropsychol Adult . February 2019. doi:10.1080/23279095.2019.1569523

123. Bell KR, Hoffman JM, Temkin NR, et al. The Effect of Telephone Counselling on Reducing PostTraumatic Symptoms After Mild Traumatic Brain Injury: A Randomised Trial. J Neurol Neurosurg Psychiatry . 2008;79(11). doi:10.1136/jnnp.2007.141762

124. Mittenberg W, Canyock EM, Condit D, Patton C. Treatment of Post-Concussion Syndrome Following Mild Head Injury. J Clin Exp Neuropsychol . 2001;23(6). doi:10.1076/jcen.23.6.829.1022

125. Gan ZS, Stein SC, Swanson R, et al. Blood Biomarkers for Traumatic Brain Injury: A Quantitative Assessment of Diagnostic and Prognostic Accuracy. Front Neurol . 2019;10. doi:10.3389/fneur.2019.00446

\section{Acknowledgement}

We would like to thank the Center for Rehabilitation of Brain Injury in Copenhagen for hosting the facilities. We would also like to thank the cases themselves, as their participation is highly valued and forms the basis for this article. Finally, we wish to thank The University College of Copenhagen for their support.

\section{Funding}

No funding was given for this study.

\section{Conflicts of Interest Statement}

The authors declare no conflicts of interest.

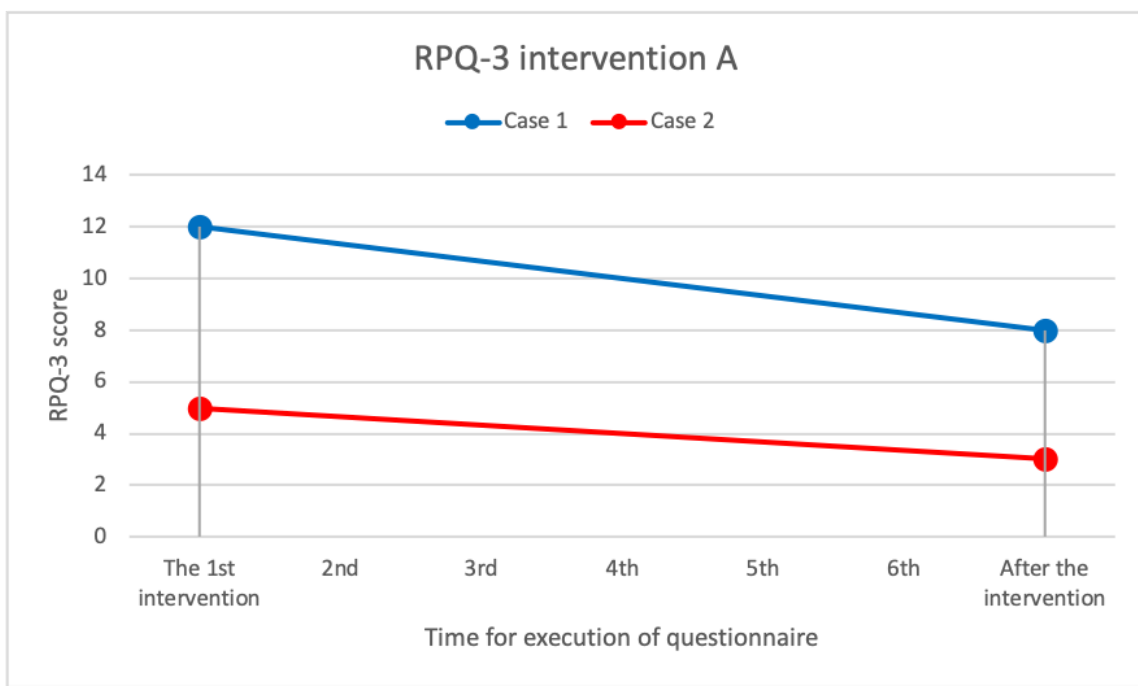

Figure 1 - Results from the Rivermead Post Concussion Questionnaire RPQ-3 score for Study A - Case 1 and 2 - conducted at first intervention and after the intervention. RPQ-3 score includes symptoms of headache, dizziness and nausea. Each symptom is scored from 0 to $4-0=$ not experienced at all, $1=$ no longer a problem, $2=$ a mild problem, $3=$ a moderate problem, $4=$ a severe problem. RPQ-3 range: $0-12$ 


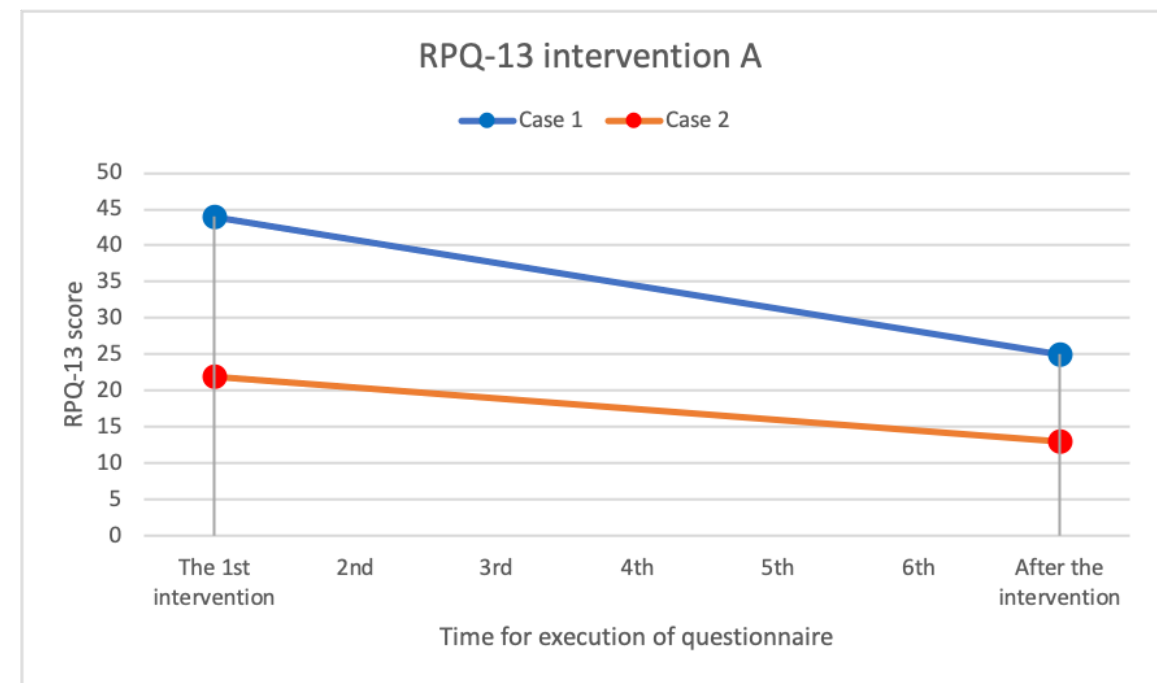

Figure 2 - Results from the Rivermead Post Concussion Questionnaire RPQ-13 score for case Study A Case 1 and 2 - conducted at first intervention and after the intervention. RPQ-13 score includes symptoms of noise sensitivity, sleep disturbance, fatigue, irritability, depression, frustration, poor memory, difficulty concentrating, reduced cognitive function, blurry vision, light sensitivity, double vision and restlessness. Each symptom is scored from 0 to $4-0=$ not experienced at all, $1=$ no longer a problem, $2=$ a mild problem, $3=$ a moderate problem, $4=$ a severe problem. $\mathrm{RPQ}-13$ range: $0-52$

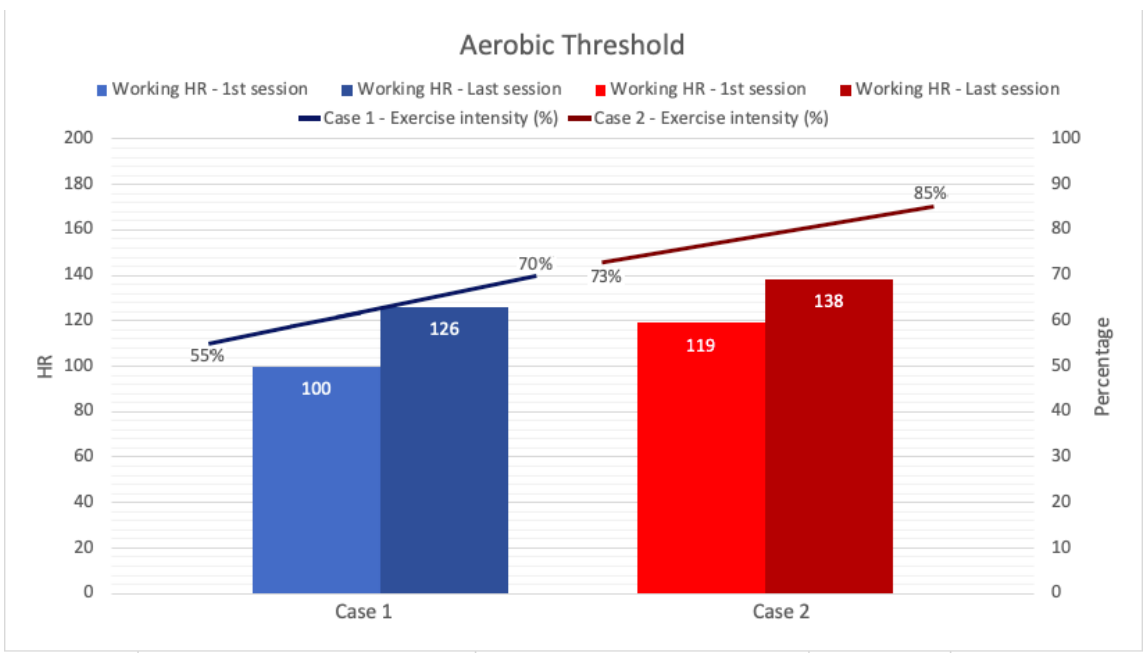

Figure 3 - Graphic illustration showing the absolute HR and the exercise intensity, expressed in percentages, first session and last session for Case 1 and 2 in Study A 


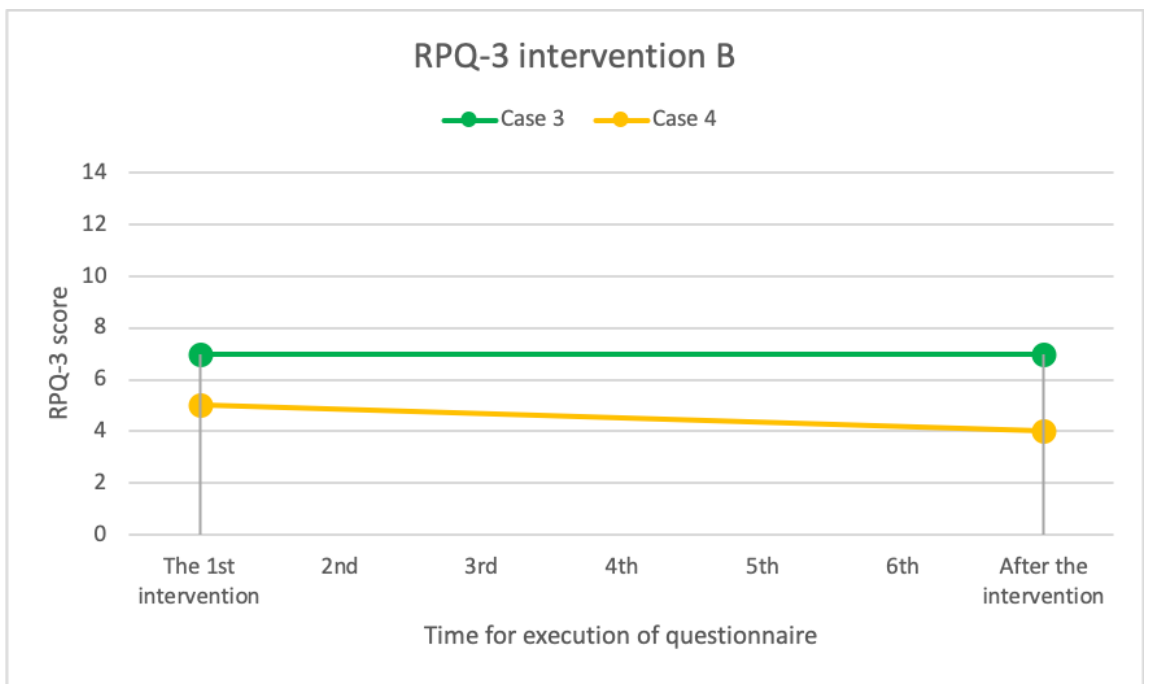

Figure 4 - Results from the Rivermead Post Concussion Questionnaire RPQ-3 score for case Study B Case 3 and 4 - conducted at first intervention and after the intervention. RPQ-3 score includes symptoms of headache, dizziness and nausea. Each symptom is scored from 0 to $4-0=$ not experienced at all, $1=$ no longer a problem, $2=$ a mild problem, $3=$ a moderate problem, $4=$ a severe problem. $R P Q-3$ range: $0-12$

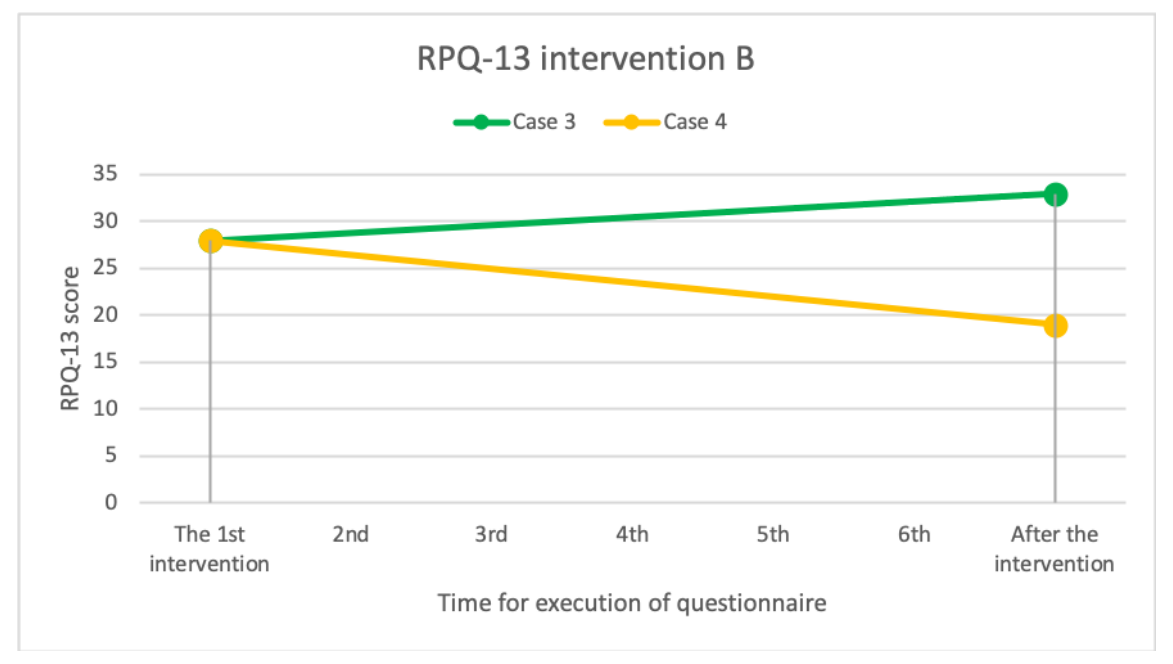

Figure 5 - Results from the Rivermead Post Concussion Questionnaire RPQ-13 score for Study B - Case 3 and 4 - conducted at first intervention and after the intervention. RPQ-13 score includes symptoms of noise sensitivity, sleep disturbance, fatigue, irritability, depression, frustration, poor memory, difficulty concentrating, reduced cognitive function, blurry vision, light sensitivity, double vision and restlessness. Each symptom is scored from 0 to $4-0=$ not experienced at all, $1=$ no longer a problem, $2=$ a mild problem, $3=$ a moderate problem, $4=$ a severe problem. $\mathrm{RPQ}-13$ range: $0-52$ 


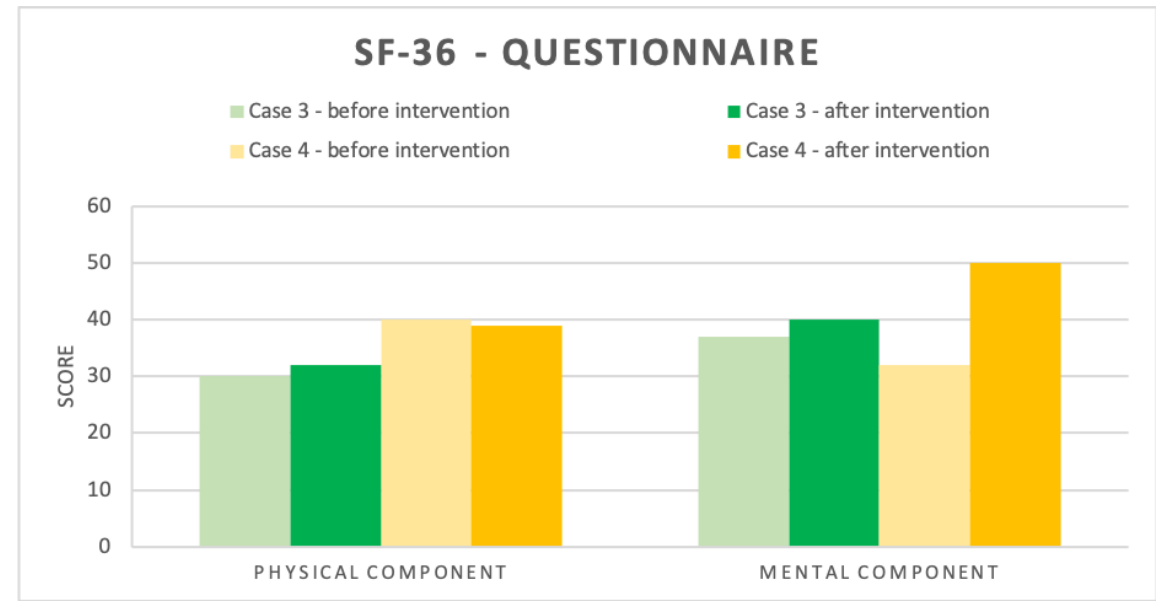

Figure 6 - Results from the SF-36 questionnaire for Study B - Case 3 and 4 - conducted before the first intervention and after the last intervention. The questionnaire is separated into mental and physical health, with higher scores signifying better quality of life

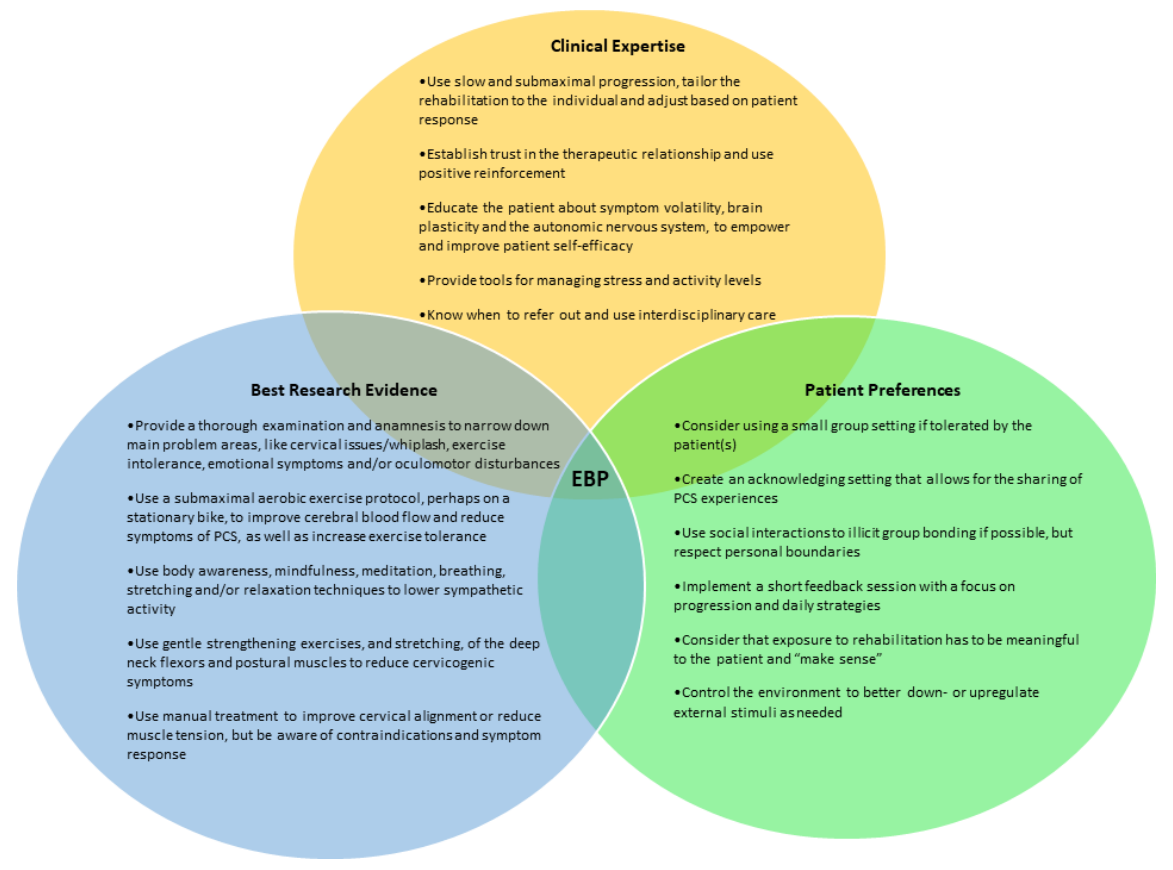

Figure 7 - The modified EBP model directed at treating PCS in the clinical practice, with bullet point suggestions encompassing the three dimensions of Best Research Evidence, Clinical Expertise and Patient Preferences 

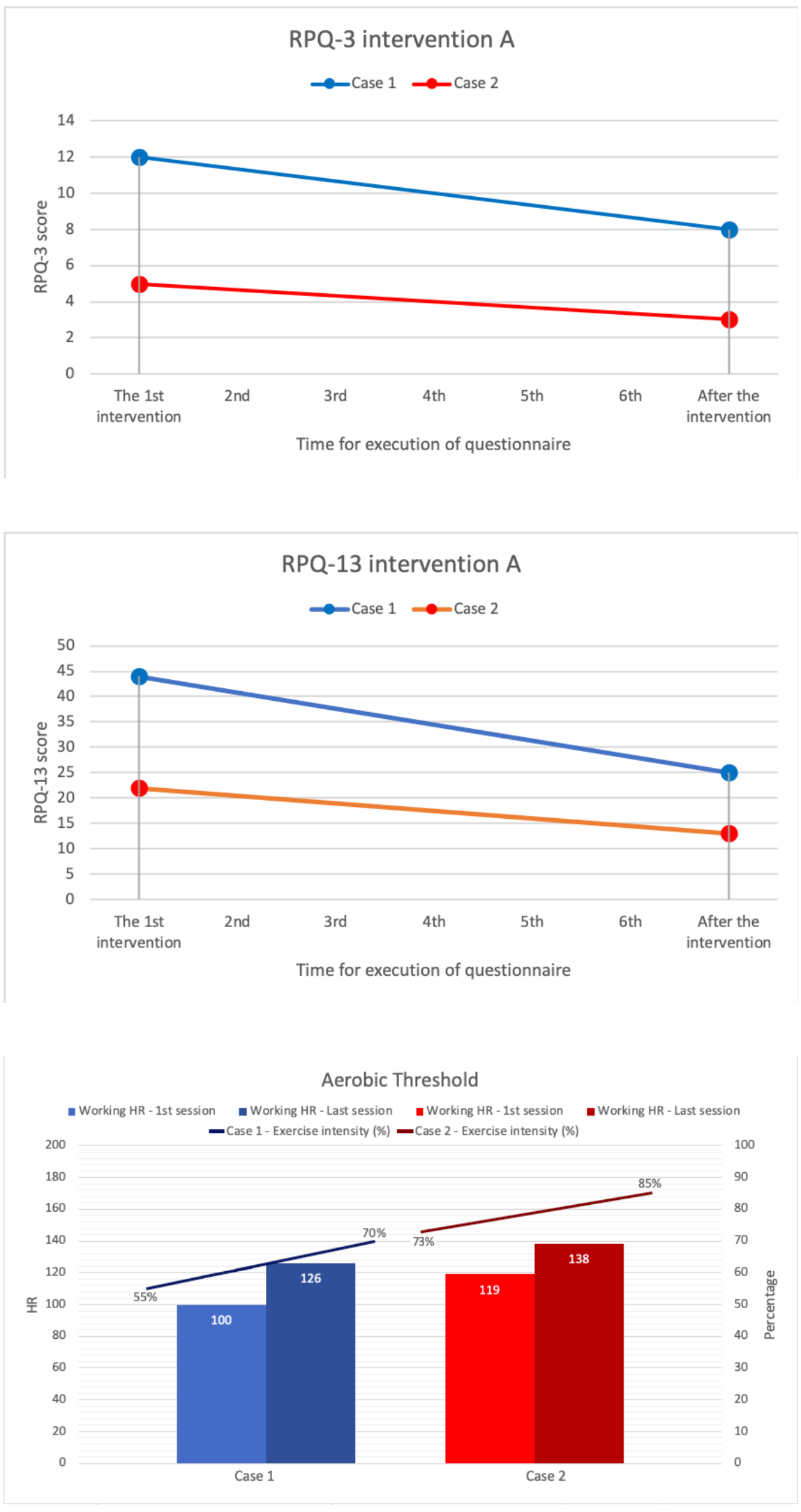

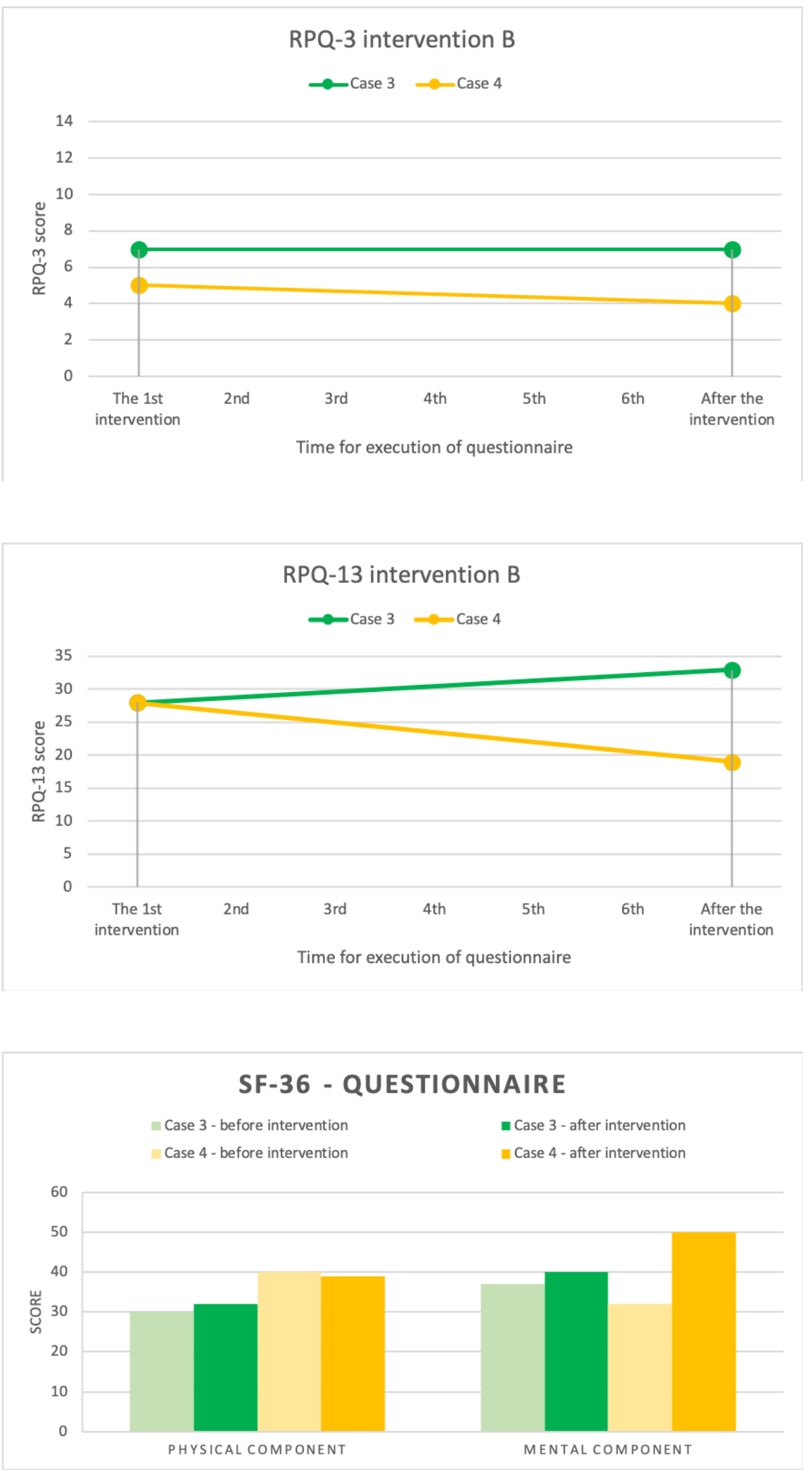


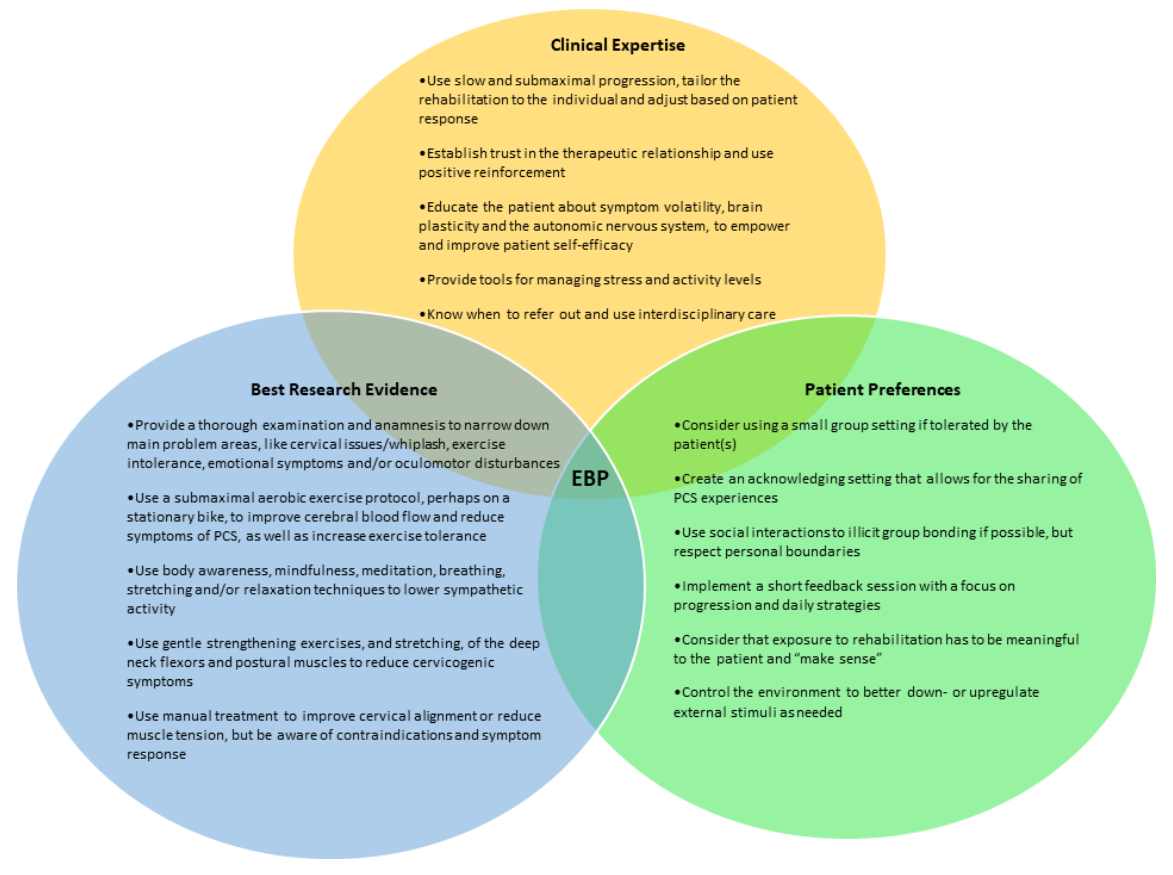

\section{Hosted file}

Table 1.docx available at https://authorea.com/users/339469/articles/465669-an-activeapproach-in-the-treatment-of-post-concussion-syndrome-evidence-based-practice-in-acollective-case-study

\section{Hosted file}

Table 2.docx available at https://authorea.com/users/339469/articles/465669-an-activeapproach-in-the-treatment-of-post-concussion-syndrome-evidence-based-practice-in-acollective-case-study

\section{Hosted file}

Table 3.docx available at https://authorea.com/users/339469/articles/465669-an-activeapproach-in-the-treatment-of-post-concussion-syndrome-evidence-based-practice-in-acollective-case-study

\section{Hosted file}

Table 4.docx available at https://authorea.com/users/339469/articles/465669-an-activeapproach-in-the-treatment-of-post-concussion-syndrome-evidence-based-practice-in-acollective-case-study 